\title{
Inhibition of carnitine palmitoyl transferase 1A- induced fatty acid oxidation suppresses cell progression in gastric cancer
}

\section{Liqiang Wang}

China-Japan Union Hospital of Jilin University https://orcid.org/0000-0002-3152-307X

\section{Changfeng Li}

China-Japan Union Hospital of Jilin University

\section{Yumei Song}

Jilin Cancer Hospital

ZhenKun Yan ( $\nabla$ yanzhenkun@jlu.edu.cn )

https://orcid.org/0000-0001-5051-8009

\section{Primary research}

Keywords: CPT1A, gastric cancer, fatty acid oxidation, prognostic, progression

Posted Date: July 10th, 2020

DOI: https://doi.org/10.21203/rs.3.rs-37846/v2

License: (c) This work is licensed under a Creative Commons Attribution 4.0 International License.

Read Full License

Version of Record: A version of this preprint was published at Archives of Biochemistry and Biophysics on December 1st, 2020. See the published version at https://doi.org/10.1016/j.abb.2020.108664. 


\title{
Inhibition of carnitine palmitoyl transferase $1 \mathrm{~A}$-induced fatty acid oxidation suppresses cell progression in gastric cancer
}

Liqiang Wang ${ }^{1}$, Changfeng $\mathrm{Li}^{1}$, Yumei Song ${ }^{2}$, ZhenKun Yan ${ }^{1, *}$

${ }^{1}$ Endoscopy Center, China-Japan Union Hospital of Jilin University, Changchun, Jilin Province, 130033, China

${ }^{2}$ Department of Thoracic Oncology, Jilin Cancer Hospital, Changchun, Jilin Province, 130033, China.

*Correspondence to: Dr ZhenKun Yan, Endoscopy Center, China-Japan Union Hospital of Jilin University, Changchun, Jilin, 130033, China. E-mail: yanzhenkun @jlu.edu.cn

\begin{abstract}
Background: Gastric cancer (GC) has a high rate of metastasis which thereason leading to death. Carnitine palmitoyltransferase 1a (CPT1A) has been reported to play a critical obstacle to various types of cancer progression, which is an attractive focus in anti-cancer therapy. However, the underlying molecular mechanisms of CPT1A involved in GC have not been clarified unclear.

Methods: To determine the expression of CPT1A in human GC tissues and cells and illustrate whether it is correlated with the clinical pathologic characteristics and prognosis in GC patients. Its roles and potential mechanisms in regulating tumor growth and invasion were evaluated by CPT1A knockdown/overexpression of GC cells in vitro. Results: Marked upregulation of CPT1A protein expression was observed in GC cells and tissues, which was associated with grade, pathological stage, lymph node metastasis and poor prognosis in patients with GC. CPT1A overexpression also promoted the proliferation, invasion, EMT process of GC cells. In addition, CPT1A upregulation activated $\mathrm{GC}$ cell $\mathrm{FAO}$ via increasing $\mathrm{NADP}^{+} / \mathrm{NADPH}$ ratio, whereas
\end{abstract}


inhibiting of FAO abolished the effects of CPT1A on GC cell proliferation and migration.

Conclusion: Our results examine that CPT1A-mediated FAO activation increases GC cell proliferation and migration, supporting that CPT1A is a useful prognostic biomarker and an attractive focus for GC.

Keywords: CPT1A; gastric cancer; fatty acid oxidation; prognostic; progression

\section{Introduction}

Globally, gastric cancer (GC) is a common malignancy and has the highest incidence and mortality in the digestive tract [1]. Although much headway has been made in terms of clinical treatment in the late years, the prognosis of GC patients is still unsatisfactory, and the 5-year survival rate is poor [2, 3]. Although several molecular markers have been proposed to detect GC diagnosis and metastasis, such as Long Noncoding RNA GMAN [4], and ER gamma [5], IRTKS [6], none has been approved for clinical use. Thus, exploring novel targets and the underlying mechanisms of GC development and metastasis is helpful for enhancing the early diagnosis of GC, even providing instructional clinical value for the treatment of metastatic GC.

Recently, the reprogramming of cellular energy metabolism, which support the unrestricted proliferation and metastatic progression of cancer cells, is widely accepted to be an emerging hallmark of cancer [7-9]. FA are fundamental cellular components and energy sources and catabolized mainly by fatty acid oxidation (FAO, also called $\beta$ oxidation), which produces more than three-fold as much ATP per mole as oxidation of carbohydrates [10-12]. Recent evidence underscores the idea that abnormal lipid metabolism mediated by FAO plays an significant part in tumor progression [13, 14]. Nieman et al. discovered that FAO-derived energy can guide ovarian cancer cells to 
metastasize to the omentum [15]. Wang et al. reported that CPT1A-induced FAO activation increased metastatic ability [16]. These findings reveals that inhibiting FAO might be the potential of strategy in cancer therapy.

Carnitine palmitoyl transferase 1 (CPT1) limits the rate-limiting step of FAO, which localizes to the outer mitochondrial membrane, and is responsible for FA transport into mitochondria for further oxidation by changing acyl-CoAs into acylcarnitines $[17,18]$. Recent studies showed that over-expression CPT1A (as a subtype of CPT1) accelerates tumor development in colorectal cancer, hepatocellular carcinoma and prostatic cancer, and is closely bound up with a poor overall survival (OS) of ovarian cancer patients [19-22]. However, the mechanistic detail of CPT1Amediated FAO activation on GC cell progression, remain obscure.

In the present study, we evaluated the CPT1A expression and attempted clarify its prognostic value and molecular mechanism in GC. Effects of CPT1A over-expression was considered to facilitate GC cells proliferation and invasion via FAO activation and conducive to an adverse outcome of GC patients. Our study supplies special insights into the potential molecular mechanisms of CPT1A involvement in FAO, and supports new tactics of molecule-targeting treatment to improve on the curative effects in GC.

\section{Materials and methods}

\section{Cell cultures and treatments}

The gastric mucosal epithelial cell line GES-1 and human GC cell lines, were acquired from the American Type Culture Collection (ATCC, Manassas, VA, USA). According to their guidelines, cells were cultured in a humid atmosphere with $5 \% \mathrm{CO}_{2}$ at $37^{\circ} \mathrm{C}$. The cell lines were tested for authenticity in 2015 using short tandem repeat (STR) genotyping and screened for mycoplasma contamination. 


\section{Lentivirus transfection}

To knockdown/ overexpress CPT1A levels, short hairpin RNA (shRNA)/fulllength targeted to human CPT1A or a non-target oligonucleotide was built into the LV3 (H1/Puro) vector. The sequence of the shRNA was 5'GGATGGGTATGGTCAAGATCT-3' and 5'-GGTGGTTTGACAAGTCGTTCA-3'. Then lentivirus was synthesized by GenePharma (Guangzhou, China). GC cells were transfected with lentivirus and treated with puromycin $(2 \mu \mathrm{g} / \mathrm{mL})$ for obtaining stably transduced cells. The cells were used for functional subsequent experiments after confirming the infection efficiency by western blot (WB) analysis.

\section{Bioinformatics analysis}

The expression of CPT1A gene in the GC and adjacent tissue were accessed on the database

of

UALCAN (http://ualcan.path.uab.edu/cgibin/TCGAExResultNew2.pl?genenam=CPT1A\&ctype =STAD), GEPIA (http://gepia.cancer-pku.cn/detail.php?gene=CPT1A), and TCGA database

(http://ualcan.path.uab.edu/cgibin/TCGAExResultNew2.pl?genenam=CPT1A\&ctype =STAD). Prognostic values of CPT1A was further assessed by using the Kaplan-Meier plotter (http://kmplot.com/analysis/index.php?p=service\&cancer=gastric).

\section{Immunohistochemical staining and analysis}

A total of $129 \mathrm{GC}$ tumor samples were stained with the CPT1A antibody (SigmaAldrich, MO). According to the ratio and intensity of positive-staining areas, two experienced pathologists conducted the CPT1A scores. Positive staining cell were 
scored as below: 0\%-5\%-“-”; 6\%-35\%-“+”; 36\%-70\%-“++”; and beyond 70\%-“+++"”. Tissue sections scored as below: negative-““-”, positive-“+, ++, +++”, strong positive“++, +++”.

\section{MTT assay}

GC cells of each group were reseeded into 96-well plates and cultured with indicated compounds for $24 \mathrm{~h}, 48 \mathrm{~h}$, and $72 \mathrm{~h}$. Then, MTT solution was added into culture medium and DMSO was utilized to dissolve formazan crystals. Removed the medium from the wells and added $100 \mu \mathrm{L}$ dimethyl sulfoxide into each well. After shaking the plate for $10 \mathrm{~s}$, the OD value of each cell at 570nm of absorbance was measured by Tecan Infnite 200 Pro microplate reader (Tecan, Switzerland).

\section{Colony formation assay}

GC cells $\left(1 \times 10^{3}\right)$ of each group were reseeded in 6 -well plates and incubated $\left(37{ }^{\circ} \mathrm{C}, 5 \% \mathrm{CO}_{2}\right)$ for 2 weeks. Then rinsed with cold PBS, colonies were fixed in methanol and stained with Giemsa. Light microscope was used to image and count.

\section{Ethynyldeoxyuridine analysis}

To detect the cell proliferation, Cell-Light ${ }^{\mathrm{TM}}$ EdU Apollo®488 (RiboBio, China) In Vitro Imaging Kit (RiboBio) was utilized according to the manufacturer's protocol after seeding cervical cancer cells in 96 -well plate for $24 \mathrm{~h}$. The result was photographed using a microscope (Olympus, Tokyo, Japan).

\section{Cell migration and invasion assay}


Transwell chamber assay was performed to detect migratory and invasive abilities of GC cells. GC cells of each group were seeded on the upper chambers with 8 - $\mu \mathrm{m}$ pore size polycarbonate membrane coated with $60 \mu \mathrm{L}$ Matrigel, and serum-free DMEM media was added to it. Then $20 \%$ fetal bovine serum/DMEM media was put into bottom chamber serving as attractant. After $24 \mathrm{~h}$ of incubation, cells were fixed with $4 \%$ formaldehyde and stained with $1 \%$ crystal violet to count the invading cells in 10 random fields by the microscope. Cell migration was investigated by transwell assay either, the only difference was no need for Matrigel. This experiment was performed 3 times for accuracy.

\section{Wound-healing assay}

Briefly, GC cells of each group were reseeded, and a linear wound was generated within the confluent monolayers by scraping the cells with sterile $10 \mu \mathrm{L}$ pipette tips. Using an inverted microscope imaged the progression of migration at $0 \mathrm{~h}, 24 \mathrm{~h}$.

\section{Western blot}

WB analysis was cultured as previously described [23]. Immunoreactive bands were visualized using ECL Western blot kit (Amersham Biosciences, Buckinghamshire, UK).

\section{Fatty acid $\beta$-oxidation and $\mathrm{NADP}^{+} / \mathrm{NADPH}$ ratio}

According to the manufacturer's instructions, using FAO Detection Kit (Genmed Scientifics, Shanghai, China) and NADP/NADPH Assay Kit (ab176724; Abcam) assayed FAO rate and the intracellular NADPH/NADP levels. 


\section{BODIPY 493/503 Staining}

GC cells $\left(4 \times 10^{3}\right)$ of each group were seeded on coverslips in DMEM plus $10 \%$ FBS. Twenty-four h post seeding cells were serum-starved for $1 \mathrm{~h}$, stained for $10 \mathrm{~min}$ with BODIPY ${ }^{\circledR}$ 493/503 (D3922, Invitrogen) and DAPI (\#4083, CST), and imaged by using an OLYMPUS BX53 microscope.

\section{Statistical procedures}

Statistical analysis was performed using SPSS 25.0 software, JMP software and GraphPad Prism 8.0. Data were carried out from at least 3 independent experiments and expressed as the mean \pm S.E.M. The chi-square test, one-way analysis of variance, and Pearson's correlation were also performed. Using the Kaplan-Meier method calculated the survival curves, and the differences were assessed by a Log-rank test. One and two asterisks indicated $P<0.05$, and $P<0.01$, respectively.

\section{Results}

\section{CPT1A is highly expressed in GC cell lines and tumor tissues}

To investigate the role of CPT1A in the development of GC, we discovered that CPT1A was markedly increased in GC tissues from the Cancer Genome Atlas (TCGA) database and GEPIA datasets (Fig. 1a and 1b). Similarly, the protein expression of CPT1A level in GC cells was remarkably higher than the expression of GES-1 (Fig. 1c). Moreover, we detected CPT1A protein expression in 45 adjacent non-tumor tissues and 129 GC tissues by IHC (Fig. 1d). IHC staining showed that the positive rate and strongly positive rates of CPT1A protein expression were only $26.7 \%(12 / 45)$ and $(15.6 \%, 7 / 45)$ in adjacent non-tumor gastric tissues, but significantly higher in GC

$(87.7 \%, 108 / 129 ; 64.3 \%, 83 / 129)(P<0.01)$ (Table 1). These results revealed that 
CPT1A displayed higher expression in GC cell lines or tumor tissues compared to their corresponding normal cells or adjacent non-tumor tissues.

\section{Upregulation of CPT1A expression is associated with pathologic features and poor} prognosis of GC patients

In TCGA database, CPT1A is upregulated in late stage, Grade 3, and LN metastatisis GC tissues compared with that in corresponding normal tissues (Fig. 2a, $2 \mathrm{~b}$, and $2 \mathrm{c}$ ). In view of the TCGA datasets, Pearson's $\chi 2$ test observed that CPT1A overexpression is correlated with Grade $3(P<0.001)$, LN metastasis $(P<0.001)$ and clinal stage (III-IV) $(P=0.001)$ (Table 2, Fig. 2d). However, no significant relations was shown between CPT1A expression and age $(P=0.475)$ or sex $(P=0.294)$.

Moreover, we predicted the prognostic value of CPT1A by comparing the OS, post progression survival (PPS), and free progression (FP) of GC patients with high or low levels of CPT1A protein. The Kaplan-Meier survival curves assessed that GC patients with high CPT1A expression had a markedly shorter OS, PPS, and FP $(P<0.05)$ (Fig. 2e). These results supported that CPT1A may be served as a potential genetic marker for human GC.

\section{CPT1A enhances the proliferation of GC cells in vitro}

To elucidate the biological functions of CPT1A in GC, we overexpressed CPT1A in SGC7901/BGC803 cells, which exhibited relatively low endogenous CPT1A levels. In addition, we knocked down CPT1A in AGS/BGC823 cells, which exhibited relatively high endogenous CPT1A levels (Fig. 3a). Subsequently, GC cells proliferation were evaluated. MTT assays shown that CPT1A overexpression or silencing markedly enhanced or inhibited cell growth in corresponding GC cells, 
respectively (Fig. 3b). EdU incorporation assays indicated that the percentage of EdUpositive cells were risen in AGS/BGC823 cells with CPT1A overexpression and were decreased in SGC7901/BGC803 cells with CPT1A knockdown (Fig. 3c). In addition, two-dimensional colony formation assays found that CPT1A overexpression or silencing markedly enhanced or impaired colony formation ability in corresponding GC cells, respectively (Fig. 3d). Therefore, these in vitro findings indicated that CPT1A enhances the proliferation of GC cells.

\section{CPT1A inhibits the migration and invasion of GC through EMT}

Based on our clinical pathological analyses supported that a positive association between CPT1A levels and LN metastatisis (Tables 2), we started to examine whether CPT1A affects GC migration and invasion. We found that CPT1A overexpression promoted the migration and invasion capability of these two cells in vitro (Fig. 4a, 4b); in contrast, knockdown of CPT1A expression levels resulted in cell wound healing inhibition (Fig. 4c), simultaneously a significant reduction of the migration and invasion capability.

Increasing evidence has demonstrated that epithelial mesenchymal transition (EMT) impels the metastatic potentials of tumor cells [24]. So, we detected the potential roles of CPT1A on EMT related marker molecules. As shown in Fig. 4d, E-cadherin expression was decreased and of Vimentin, and Snail were increased in GC cells, which downregulated CPT1A expression. In contrast, overexpression of CPT1A had reverse functions on EMT related proteins. These results indicated that CPT1A enhances the migration and invasion capability of GC through activating EMT.

\section{CPT1A activates FAO in GC cells}


Accumulation of lipid is necessary for tumor cells to satisfy the high-energy demand of their rapid proliferation and metastasis. We next appraised alterations in lipid accumulation using BODIPY 493/503 staining, and indicated that CPT1A silencing markedly promoted lipid droplet accumulation in SGC7901/BGC803 cells, whereas CPT1A overexpression suppressed it in AGS/BGC823 (Fig. 5A). Evaluation of changes in FAO level and $\mathrm{NADP}^{+} / \mathrm{NADPH}$ ratio also revealed, $\mathrm{CPT} 1 \mathrm{~A}$ silencing significantly suppressed FAO level and NADP ${ }^{+} / \mathrm{NADPH}$ ratio in SGC7901/BGC803 cells. Conversely, the results were opposite (Fig. $5 \mathrm{~b}$ and $5 \mathrm{c}$ ). These data indicated that CPT1A has involved in lipid accumulation of GC cells. Furthermore, we focus on the regulatory effects of CPT1A on key enzymes (ACSL1, ACSL5, and ACC1) involved in FAO. Western blot showed that CPT1A silencing could significantly reduced the expression levels of key enzymes, whereas CPT1A overexpression enhanced the expression levels (Fig. 5d). These results indicated that CPT1A induces FAO in GC cells.

\section{CPT1A promotes the tumor progression of gastric cancer cells by regulating FAO}

To further explore the function of CPT1A in GC cells, we treated with the FAO inhibitors-Etomoxir (ETX) in CPT1A-overexpressed GC cells, and measured cell proliferation and migration. We observed that ETX significantly decreased cell proliferative capacity in CPT1A-overexpressed GC cells in comparison with control cells (Fig. 6a). Colony formation assays showed a reduction in both the size and number of colonies in ETX-treated cells (Fig. 6b). Similarly, inhibition of FAO markedly inhibited cell migration capacity as determined by Transwell (Fig. 6c). In addition, ETX significantly reduced $\mathrm{FAO}$ and $\mathrm{NADP}^{+} / \mathrm{NADPH}$ ratio (Fig. 6d and 6e). Western blot shown that ETX reversed the CPT1A-induced upregulation of Vimentin, ACSL1, and 
ACC1 and downregulation of Ecadherin (Fig. 6f). These results expounded that CPT1A regulates cell growth, migration, and the EMT process in a manner dependent on the FAO pathway.

\section{Discussion}

Previous studies reported that CPT1A serves a key role in the development of numerous cancers, and contributes to tumor growth and metastasis [25-27]. Xiong et al. found that CPT1A knockdown attenuates invasion and lymphangiogenesis through the regulation of VEGF signaling in breast cancer cell [28]. A recent study demonstrated that CPT1A essential for HCC cell survival, and Diet-induced hepatic steatosis activates Ras to promote hepatocarcinogenesis via CPT1A [29]. Furthermore, the PGC1 $\alpha / \mathrm{CEBPB} / \mathrm{CPT} 1 \mathrm{~A}$ signaling axis advances the resistance to radiation in NPC via activating FAO [30]. Despite these accumulating evidence on the role of CPT1A in cancer progression, whether CPT1A could mediate GC growth and invasion, and by which underlying mechanisms, remain unclear. The present study aimed therefore to clarify the role of CPT1A in GC growth and invasion.

Shi et al. expounded that elevated CPT1A expression is closely bound up with the shorter survival of AML [31]. In the present study, CPT1A was found to be frequently upregulated in GC using bioinformatics and experimental analysis (Fig. 1, 2). IHC shown that the positive stain intensity of CPT1A was mostly expressed in GC tissues, and weak staining was expressed in adjacent non-tumor tissue (Fig. 1d, e). More interestingly, the up-regulation level of CPT1A was closely related to some clinical pathological factors (stage, grade, and LN metastasis) $(P<0.01)$ (Fig. 2d). However, no differences were found with age, sex, or tumor size (Table 2). In particular, we found that GC patients exhibiting high CPT1A expression had poorerm OS, PPS and FP than 
patients with low expression by Kaplan-Meier Plotter datasets (Fig. 2e). Then, we performed survival analyses to evaluate that CPT1A is a prognostic marker for clinical outcomes. As a result, CPT1A may function as an important indicator during the germination and progression of GC.

Previous studies reported that CPT1A is abundant in advanced prostate cancer (PCa), and CPT1A knockdown decreased clonogenic growth and invasion in PCa cell [32]. Wang et al. demonstrated that the molecular functions of CPT1A as a succinyl transferase by targeting S100A10 and activating its succinylation, which promotes cancer invasion and metastasis [33]. Consistent with these findings, our reasearch have confirmed an important mechanism of CPT1A in GC proliferation. Then, we appraised the variations of cell migration and invasion in GC cells transfect with or without CPT1A, and found that silencing of CPT1A results in cell migration and invasion arrested, simultaneously overexpression of CPT1A exposed the contrary results. Besides, we assessed the variations of EMT-related marker proteins, which were in charge of the transition of the cell from the epithelium-like shape to the mesenchymal phenotype and their up/down-regulation would lead to cell EMT process [34, 35]. As expounded by our data, silencing of CPT1A lead to down-regulate expression of the Vimentin, Snail and increased E-cadherin. Conversely, the results were opposite, in accordance with previous fndings [28]. These results supported that CPT1A can influence the cancer cell progression by regulating the expression of EMT marker proteins, thus affected GC cell process.

Increasing evidence has placed that a novel emphasis on FAO as an important metabolic crux in cancer, which is the main approach for the degradation of FA and enhances the production of NADPH and ATP [36]. For instance, advanced FAO supplies glioblastoma cells metabolic malleability to accommodate to its rich nutrient 
microenvironment [37]. And Wang et al. indicated a distinctive mechanism by which IL-17A mediates FAO stimulates tumor angiogenesis of endothelial cells by enhancing endothelial mitochondrial respiration [38]. In the present study, we observed that of CPT1A knockdown increased lipid droplet accumulation, decreases FAO and $\mathrm{NADP}^{+} / \mathrm{NADPH}$ ratio in GC cells, and vice versa (Fig. 5a-c). Consistently, we found that the expression ratios of FAO-related proteins were decreased/increased following cell treatment with CPT1A down-regulate/up-regulate (Fig. 5d). Unsurprisingly, we confirmed that CPT1A could increase FAO level in GC cell by NADPH homeostasis.

A previous reasearch identified that targeting with metabolic reprogramming of cancer cells might be a potential therapeutic strategy and CPT1A has been indicated as a target of FA metabolism [39]. Wang et al. CPT1A-mediated FAO accelerates colorectal cancer cell metastasis by avoiding anoikis [16]. Hence, we hypothesized that CPT1A could be involved in GC proliferation and invasion through the FAO. To do so, the effects of Etomoxir (an inhibitor of FAO) on AGS/BGC823 cells with CPT1A overexpression was assessed. A few studies have reported that Etomoxir had an influence on CPT1A expression which could irreversibly inhibit the function of CPT1 [40]. Although recently some studies identified an off-target effect of etomoxir [41, 42], still we could see the FAO inhibition of Etomoxir in research. Consistently, the FAO inhibition of etomoxir could be validated by decreased FAO and NADPH $\mathrm{NADP}^{+} / \mathrm{NADPH}$ ratio in the present study (Fig. 6d-e). In addition, our results showed that FAO inhibition by Etomoxir could suppress growth, migration and EMT process both in CPT1A overexpression GC cells (Fig. 6a-c, 6f). Taken together, these findings indicated that CPT1A stimulates GC growth and migration by activating FAO.

\section{Conclusions}


Through this study, our data clearly identified that FAO confers tumor progression via a CPT1A-mediated mechanism in GC (Fig. 7). The results confirmed that CPT1A expression was signifcantly elevated in GC tissues and cells, and that high expression of CPT1A was closely related with Grade, LN metastasis, and clinal stage and poor prognosis in GC patients. Moreover, CPT1A overexpression have facilitated GC cell progression, probably by activating FAO. However, future multi-center studies with a larger sample size are required to verify that CPT1A could be a novel prognostic and potential therapeutic target for patients with GC.

\begin{abstract}
Abbreviations
GC: gastric cancer; FAO: fatty acid oxidation, also called $\beta$-oxidation; CPT1: Carnitine palmitoyl transferase 1; OS: overall survival; STR: short tandem repeat; shRNA: short hairpin RNA;PPS: progression survival; FP: free progression; ETX: Etomoxir; EMT: epithelial mesenchymal transition; PCa: prostate cancer.
\end{abstract}

\title{
Acknowledgement
}

We wish to acknowledge that the project was supported by ChinaJapan Union Hospital of Jilin University.

\section{Authors' contributions}

Study design: ZK Y and LQ W. Study conduct: SJ, HJ and LJ. Data collection: LQ W, CF L and YM S. Data analysis: LQ W, CF L and YM S. Data interpretation: YM S and ZK Y. Drafting manuscript: LQ W and ZK Y.

Disclosure statement: All the authors declare that they have no conflicts of interest. 


\section{Funding}

Not applicable.

\section{Availability of data and materials}

Datasets used and/or analyzed data are available from the corresponding author upon reasonable request.

\section{Consent for publication}

Not applicable.

\section{Competing interests}

The authors declare that they no competing interests.

\section{Author details}

${ }^{1}$ Endoscopy Center, China-Japan Union Hospital of Jilin University, Changchun, Jilin, 130000, China.

${ }^{2}$ Department of Thoracic Oncology, Tumor Hospital of Jilin Province, Changchun, Jilin, 130000, China.

\section{Reference}

1. Luo Z, Rong Z, Zhang J, et al. Circular RNA circCCDC9 acts as a miR-6792-3p sponge to suppress the progression of gastric cancer through regulating CAV1 expression. Mol Cancer. 2020; 19(1): 86. 
2. Tian S, Jiang R, Madden NA, et al. Survival outcomes in patients with gastric and gastroesophageal junction adenocarcinomas treated with perioperative chemotherapy with or without preoperative radiotherapy. Cancer. 2020; 126(1): 37-45.

3. Lin JX, Xie XS, Weng XF, et al. UFM1 suppresses invasive activities of gastric cancer cells by attenuating the expression of PDK1 through PI3K/AKT signaling. $J$ Exp Clin Cancer Res. 2019; 38(1): 410.

4. Zhuo W, Liu Y, Li S, et al. Long Noncoding RNA GMAN, Up-regulated in gastric cancer tissues, is associated with metastasis in patients and promotes translation of Ephrin A1 by competitively binding GMAN-AS. Gastroenterology. 2019; 156: 676691.

5. Kang MH, Choi H, Oshima M, et al. Estrogen-related receptor gamma functions as a tumor suppressor in gastric cancer. Nat Commun. 2018; 9: 1920.

6. Huang LY, Wang X, Cui XF, et al. IRTKS is correlated with progression and survival time of patients with gastric cancer. Gut. 2018; 67: 1400-1409.

7. Shang C, Wang W, Liao Y, et al. LNMICC promotes nodal metastasis of cervical cancer by reprogramming fatty acid metabolism. Cancer Res. 2018; 78(4): 877-890.

8. Warburg O. On the origin of cancer cells. Science. 1956; 123(3191): 309-314.

9. Chen X, Wei S, Ma Y, et al. Quantitative proteomics analysis identifies mitochondria as therapeutic targets of multidrug-resistance in ovarian cancer. Theranostics. 2014; 4(12): 1164-1175.

10. Kastaniotis AJ, Autio KJ, Keratar JM, et al. Mitochondrial fatty acid synthesis, fatty acids and mitochondrial physiology. Biochim Biophys Acta. 2017; 1862(1): 39-48.

11. Houten SM, Wanders RJ. A general introduction to the biochemistry of mitochondrial fatty acid beta-oxidation. J Inherit Metab Dis. 2010; 33: 469-477.

12. Houten SM, Violante S, Ventura FV, Wanders RJ. The biochemistry and physiology 
of mitochondrial fatty acid betaoxidation and its genetic disorders. Annu Rev Physiol. 2016; 78: 23-44.

13. Beloribi-Djefaflia S, Vasseur S, Guillaumond F. Lipid metabolic reprogramming in cancer cells. Oncogenesis. 2016; 5: e189.

14. Carracedo A, Cantley LC, Pandolfi PP. Cancer metabolism: fatty acid oxidation in the limelight. Nat Rev Cancer. 2013; 13: 227-232.

15. Nieman KM, Kenny HA, Penicka CV, et al. Adipocytes promote ovarian cancer metastasis and provide energy for rapid tumor growth. Nat Med. 2011; 17: 1498-1503. 16. Wang YN, Zeng ZL, Lu J, et al. CPT1A-mediated fatty acid oxidation promotes colorectal cancer cell metastasis by inhibiting anoikis. Oncogene. 2018; 37(46): 60256040.

17. Zammit VA. Carnitine palmitoyltransferase 1: central to cell function. IUBMB Life. 2008; 60: 347-354.

18. Setoyama D, Fujimura Y, Miura D. Metabolomics reveals that carnitine palmitoyltransferase-1 is a novel target for oxidative inactivation in human cells. Genes Cells. 2013; 18: 1107-1119.

19. Schreurs M, Kuipers F, van der Leij FR. Regulatory enzymes of mitochondrial betaoxidation as targets for treatment of the metabolic syndrome. Obes Rev. 2010; 11(5): $380-388$

20. Shao H, Mohamed EM, Xu GG, et al. Carnitine palmitoyltransferase 1A functions to repress FoxO transcription factors to allow cell cycle progression in ovarian cancer. Oncotarget. 2016; 7(4): 3832-3846.

21. Schlaepfer IR, Rider L, Rodrigues LU, et al. Lipid catabolism via CPT1 as a therapeutic target for prostate cancer. Mol Cancer Ther. 2014; 13: 2361-2371.

22. Xu A, Wang B, Fu J, et al. Diet-induced hepatic steatosis activates Ras to promote 
hepatocarcinogenesis via CPT1alpha. Cancer Lett. 2019; 442: 40-52.

23. Yang $\mathrm{Y}, \mathrm{Wu} \mathrm{Q}, \mathrm{Li} \mathrm{N}$, et al. Upregulation of Tiam1 contributes to cervical cancer disease progression and indicates poor survival outcome. Hum Pathol. 2018; 75: 179188.

24. Liu WG, Xu Q. Upregulation of circHIPK3 promotes the progression of gastric cancer via Wnt/ $\beta$-catenin pathway and indicates a poor prognosis. Eur Rev Med Pharmacol Sci. 2019; 23(18): 7905-7912.

25. Qu Q, Zeng F, Liu X, Wang QJ, Deng F. Fatty acid oxidation and carnitine palmitoyltransferase I: emerging therapeutic targets in cancer. Cell Death Dis. 2016; 7: e2226.

26. Gatza ML, Silva GO, Parker JS, Fan C, Perou CM. An integrated genomics approach identifies drivers of proliferation in luminalsubtype human breast cancer. Nat Genet. 2014; 46: 1051-1059.

27. Ricciardi MR, Mirabilii S, Allegretti M, et al. Targeting the leukemia cell metabolism by the CPT1a inhibition: functional preclinical effects in leukemias. Blood. 2015; 126: 1925-1929.

28. Xiong Y, Liu Z, Zhao X, CPT1A regulates breast cancer-associated lymphangiogenesis via VEGF signaling. Biomed Pharmacother. 2018;106:1-7.

29. Xu A, Wang B, Fu J, et al. Diet-induced hepatic steatosis activates Ras to promote hepatocarcinogenesis via CPT1 $\alpha$. Cancer Lett. 2019; 442: 40-52.

30. $\mathrm{Du} \mathrm{Q}$, Tan $\mathrm{Z}$, Shi $\mathrm{F}$, et al. PGC1 $\alpha / \mathrm{CEBPB} / \mathrm{CPT} 1 \mathrm{~A}$ axis promotes radiation resistance of nasopharyngeal carcinoma through activating fatty acid oxidation. Cancer Sci. 2019; 110(6): 2050-2062.

31. Shi J, Fu H, Jia Z, et al. High Expression of CPT1A Predicts Adverse Outcomes: A Potential Therapeutic Target for Acute Myeloid Leukemia. EBioMedicine. 2016; 14: 
$55-64$

32. Flaig TW, Salzmann-Sullivan M, Su LJ, et al. Lipid catabolism inhibition sensitizes prostate cancer cells to antiandrogen blockade. Oncotarget. 2017; 8(34): 56051-56065 33. Wang C, Zhang C, Li X, et al. CPT1A-mediated succinylation of S100A10 increases human gastric cancer invasion. J Cell Mol Med. 2019; 23(1): 293-305

34. Li N, Piao J, Wang X, et al. Paip1 indicated poor prognosis in cervical cancer and promoted cervical carcinogenesis. Cancer Res Treat. 2019; 51(4): 1653-1665

35. Cook DP, Vanderhyden BC. Context specificity of the EMT transcriptional response. Nat Commun. 2020; 11(1): 2142.

36. Carracedo A, Cantley LC, Pandolfi PP. Cancer metabolism: fatty acid oxidation in the limelight. Nat Rev Cancer. 2013; 13: 227-232.

37. Kant S, Kesarwani P, Prabhu A, et al. Enhanced fatty acid oxidation provides glioblastoma cells metabolic plasticity to accommodate to its dynamic nutrient microenvironment. Cell Death Dis. 2020; 11(4): 253.

38. Wang R, Lou X, Feng G, et al. IL-17A-stimulated endothelial fatty acid $\beta$-oxidation promotes tumor angiogenesis. Life Sci. 2019; 229: 46-56.

39. Vander Heiden MG. Targeting cancer metabolism: a therapeutic window opens. Nat Rev Drug Discovery. 2011; 10: 671-684.

40. Schlaepfer IR, Rider L, Rodrigues LU, et al. Lipid catabolism via CPT1 as a therapeutic target for prostate cancer. Mol Cancer Ther. 2014; 13(10): 2361-2371.

41. Raud B, Roy DG, Divakaruni AS, et al. Etomoxir actions on regulatory and memory $\mathrm{T}$ cells are independent of Cptla-mediated fatty acid oxidation. Cell Metab. 2018; 28(3): 504-515.

42. van Vuurden DG, Aronica E, Hulleman E, et al. Pre-B-cell leukemia homeobox interacting protein 1 is overexpressed in astrocytoma and promotes tumor cell growth 
and migration. Neuro Oncol. 2014; 16(7): 946-959.

\section{Figure Legend}

Fig 1. CPT1A was highly expressed in GC cell lines and tumor tissues. (a-b). Box plots derived from gene expression data in Oncomine (a) and GEPIA (b) comparing the mRNA expression of CPT1A in normal and GC tissue $(P<0.05)$. (c). The expression of CPT1A protein in a normal gastric cell line (GES-1) and various GC cancer cell lines was examined by Western blot analysis. (d). Percentage of CPT1A IHC in GC and adjacent normal tissues on tissue microarray $(\times 200)$. (e). IHC of CPT1A in GC tissues with different staining intensity $(\times 200)$.

Fig 2. High expression of CPT1A correlates with poor prognosis of GC patients.

(a-c). Analysis of CPT1A expression in matched normal and different stage (a), grade (b), and nodal metastasis (c) GC tissues in the TCGA data set. (d). The high expression level of CPT1A protein was significantly related to clinical stage $(P=0.0004)$, Grade $(P=0.0034)$ and LN metastasis $(P=0.000)$. (e). High CPT1A mRNA levels were associated with shorter OS, PPS, and FP in GC patients.

Fig 3. Cell proliferation is accelerated and attenuated with enhancement and suppression of CPT1A in human GC cells. (a). WB analysised was onfirmed the protein expression of CPT1A in the constructed GC cells. $\beta$-Actin was used as a loading control. (b-d). Cell proliferation was examined by MTT (b), EdU (c) and colony formation (d) in the constructed cells. Data are represented as mean \pm standard error of mean of at least three independent experiments, ${ }^{*} P<0.05 ; * * P<0.01$.

Fig 4. CPT1A enhanced cell migration and invasion in human GC cells. (a). Monolayer wound healing assay was explored the migration abilities of GC cells with CPT1A overexpressed or silenced $(\times 100)$. (b-c). Transwell cell migration (b) and invasion (c) 
assays tested that CPT1A promotes both cell migratory and invasive capacities in GC cells. The stained cells were counted from four selected fields randomly. Representative images and quantitative results of cell migration and invasion were shown $(\times 100)$. (d). Western blot analysis of EMT marker (E-cadherin, Vimentin and Snail) in indicated cells with overexpressed or silenced of CPT1A. $\beta$-Actin was used as a loading control. Data are represented as mean \pm standard error of mean of at least three independent experiments, $* P<0.05 ; * * P<0.01$.

Fig 5. CPT1A enhanced the fatty acid $\beta$-oxidation (FAO) in human GC cells. (a). To indicate fat droplet deposit by Bodipy 493/503 staining in CPT1A-treated GC cells $(\times 200 ; \times 400) .(b-c)$. The level of FAO (b) and The NADPH/NADP ${ }^{+}$ratio (c) in CPT1Asilenced and CPT1A-overexpressed GC cells. (d). Western blot analysis of FAO marker (ACSL1, ACLS5, ACC1) in indicated cells with overexpressed or silenced of CPT1A. $\beta$-Actin was used as a loading control. Data are represented as mean \pm standard error of mean of at least three independent experiments, $* P<0.05 ; * * P<0.01$.

Fig 6. Inhibition of FAO attenuates CPT1A-induced proliferation and migration in GC cells. (a). Cell viability of CPT1A-overexpressed GC cells treated by Etomoxir (ETX) for 24, 48 and $72 \mathrm{~h}$ was measured by MTT. (b). Representative images (left) and quantification (right) of colony formation assay for the ETX-inhibited effects in CPT1A-overexpressed GC cells. (c). Representative images (left) and quantification (right) of Migration assay for the ETX-inhibited effects in CPT1A-overexpressed GC cells. (d-e). The level of FAO (d) and The NADPH/NADP ${ }^{+}$ratio (e) for the ETXinhibited effects in CPT1A-overexpressed GC cells. D. E-cadherin, Vimentin, ACSL1 and ACC1 were determined by western blot after exposure to ETX for $48 \mathrm{~h}$ in CPT1Aoverexpressed GC cells. $\beta$-Actin was used as a loading control. Data are represented as 
mean \pm standard error of mean of at least three independent experiments, $* P<0.05$; $* * P<0.01$

Fig 7. Schematic pathways of CPT1A mediated growth and EMT in breast cancer cells due to increased mitochondrial FAO metabolism. 


\section{Figures}

a

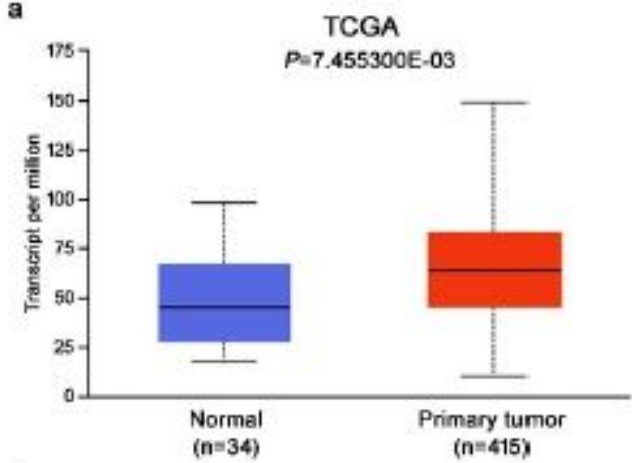

c

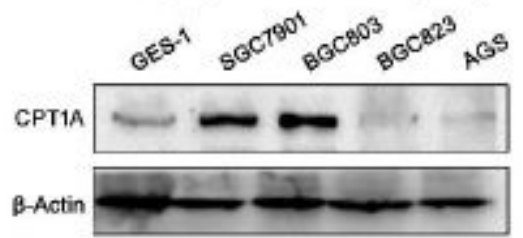

d

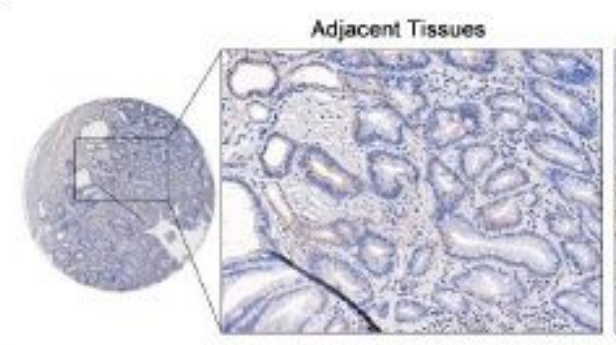

$(-)$

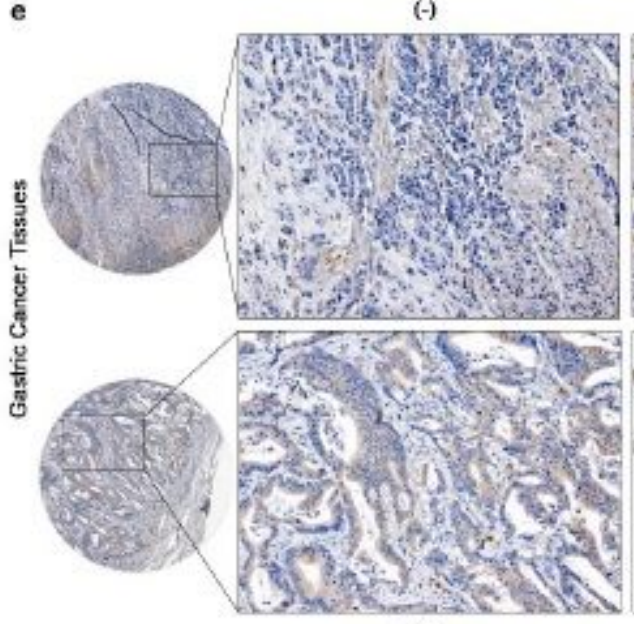

$(++)$

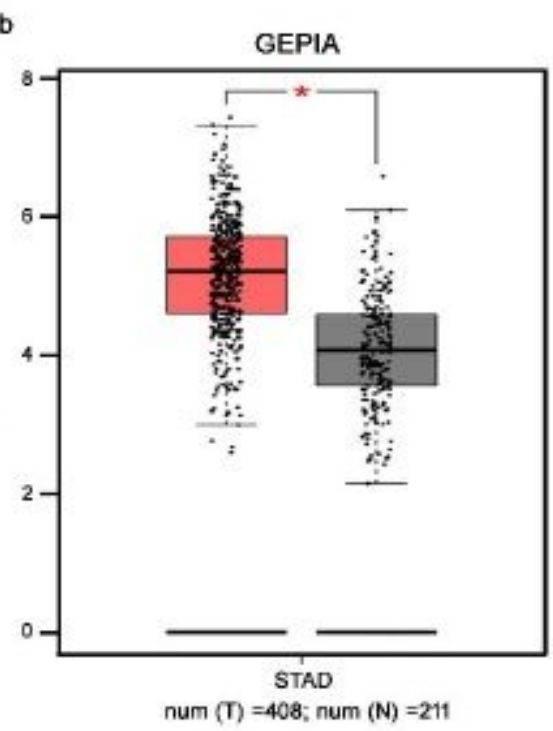

Gastric Cancer Tissues

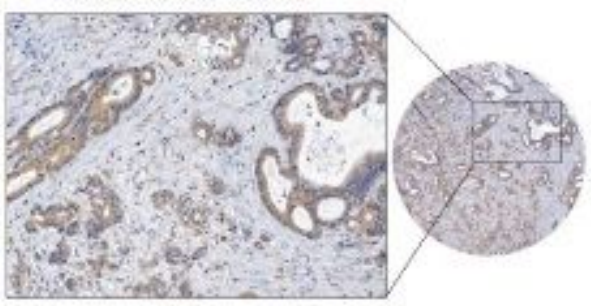

(+)

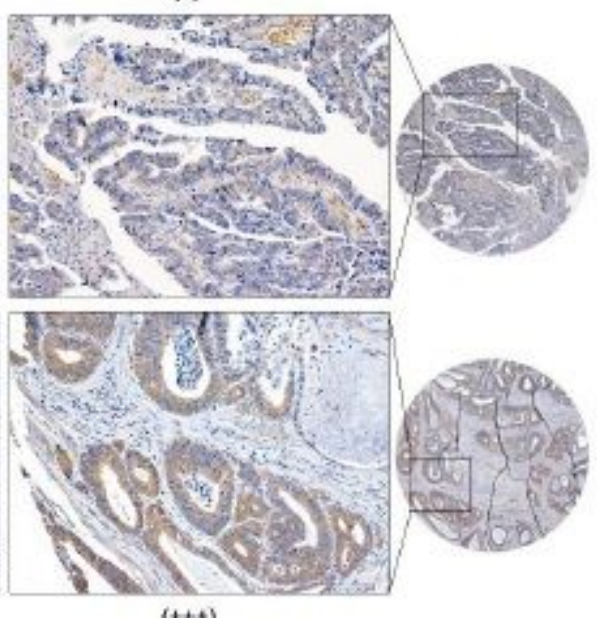

$(++t)$

Figure 1

CPT1A was highly expressed in GC cell lines and tumor tissues. (a-b). Box plots derived from gene expression data in Oncomine (a) and GEPIA (b) comparing the mRNA expression of CPT1A in normal and GC tissue $(P<0.05)$. (c). The expression of CPT1A protein in a normal gastric cell line (GES-1) and various GC cancer cell lines was examined by Western blot analysis. (d). Percentage of CPT1A IHC in GC and 
adjacent normal tissues on tissue microarray $(\times 200)$. (e). IHC of CPT1A in GC tissues with different staining intensity $(\times 200)$.
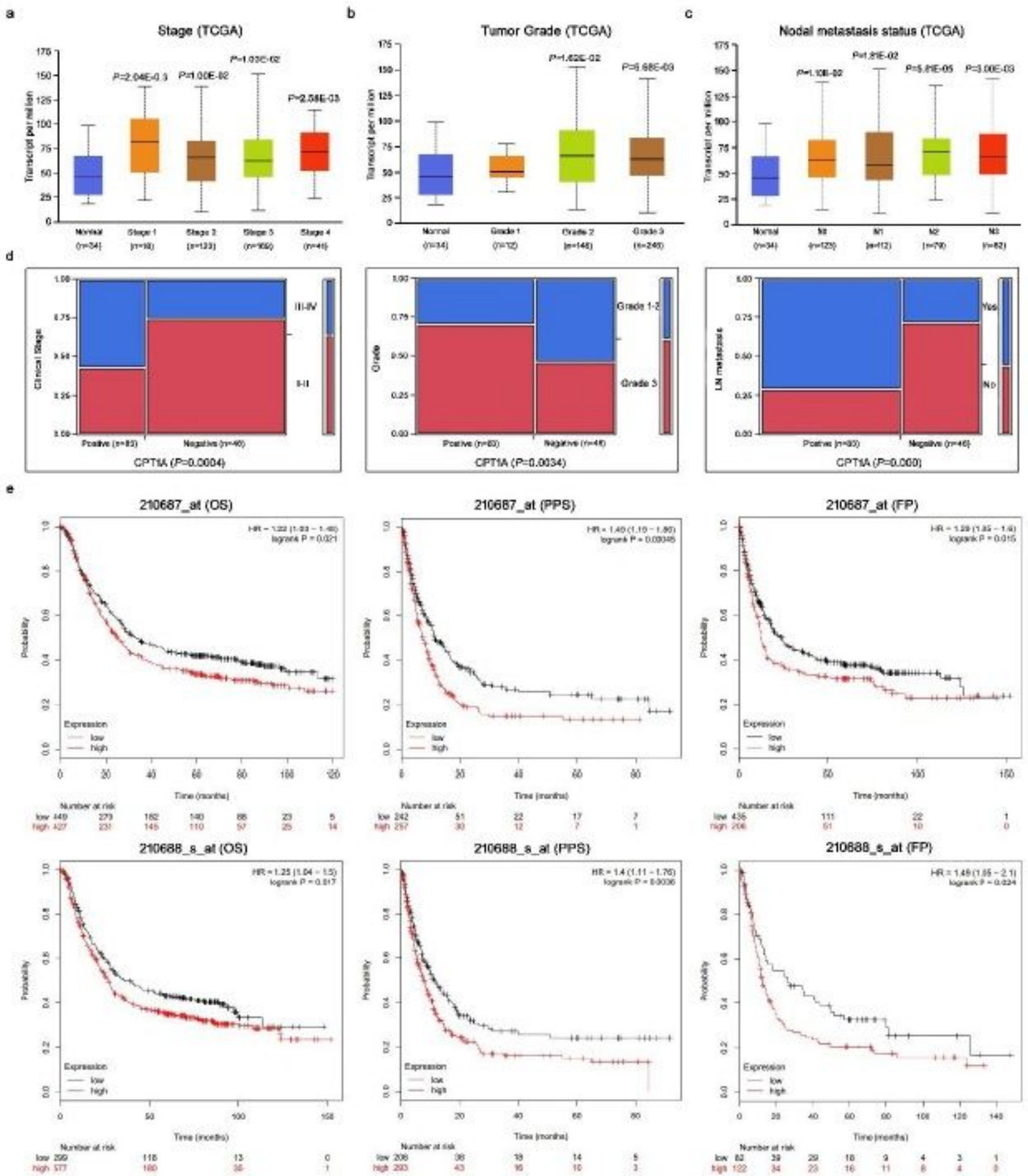

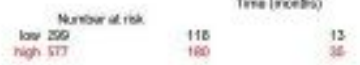

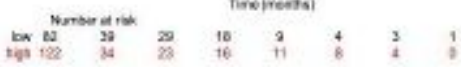

\section{Figure 2}

High expression of CPT1A correlates with poor prognosis of GC patients. (a-c). Analysis of CPT1A expression in matched normal and different stage (a), grade (b), and nodal metastasis (c) GC tissues in the TCGA data set. (d). The high expression level of CPT1A protein was significantly related to clinical 
stage $(P=0.0004)$, Grade $(P=0.0034)$ and LN metastasis $(P=0.000)$. (e). High CPT1A mRNA levels were associated with shorter OS, PPS, and FP in GC patients.
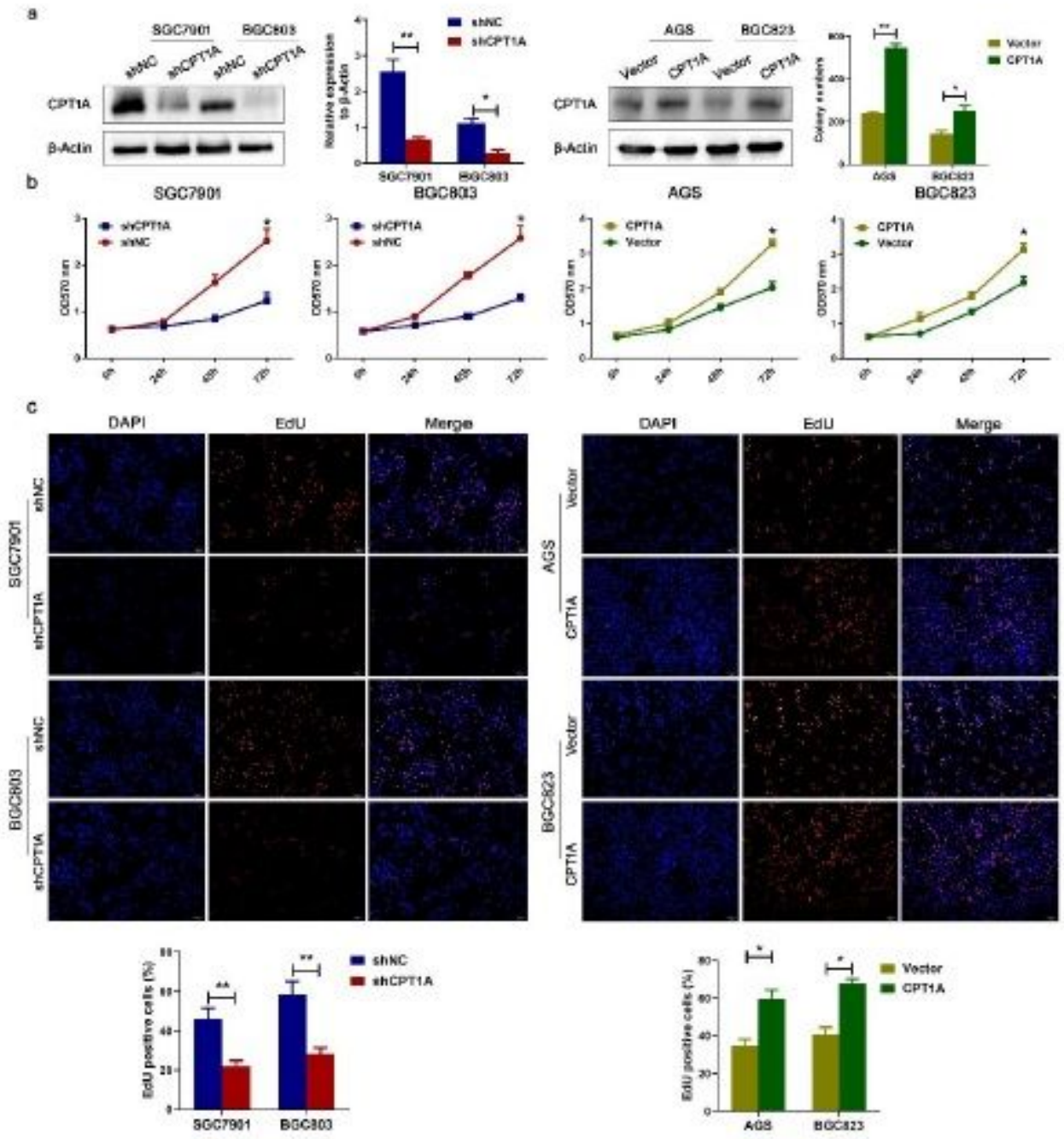

d

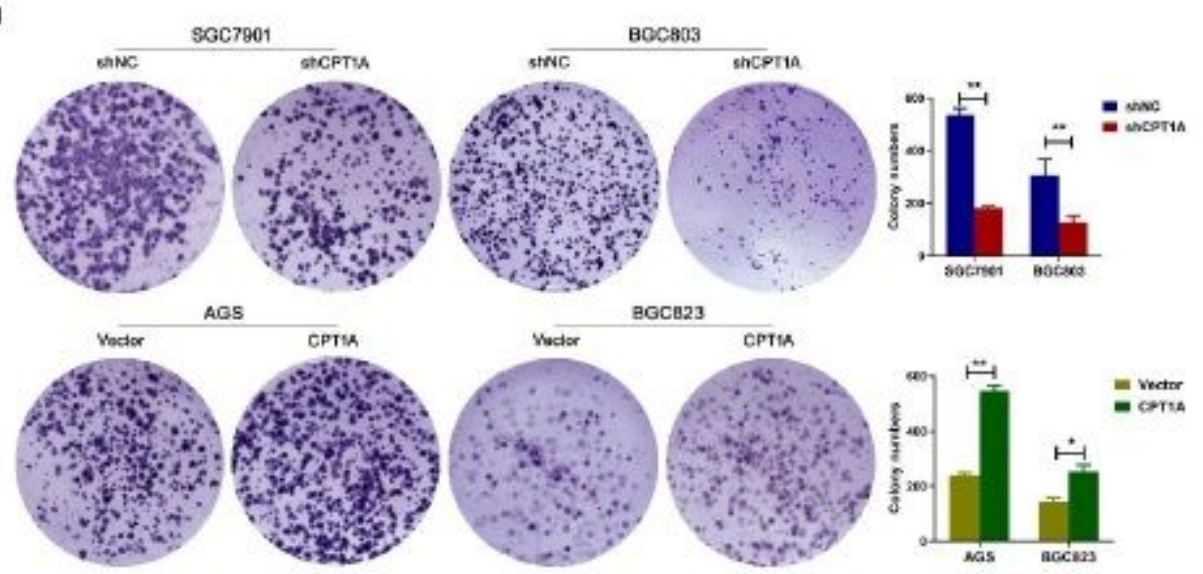

\section{Figure 3}

Cell proliferation is accelerated and attenuated with enhancement and suppression of CPT1A in human GC cells. (a). WB analysised was onfirmed the protein expression of CPT1A in the constructed GC cells. $\beta$ Actin was used as a loading control. (b-d). Cell proliferation was examined by MTT (b), EdU (c) and 
colony formation (d) in the constructed cells. Data are represented as mean \pm standard error of mean of at least three independent experiments, ${ }^{*} \mathrm{P}<0.05 ;{ }^{* *} \mathrm{P}<0.01$.

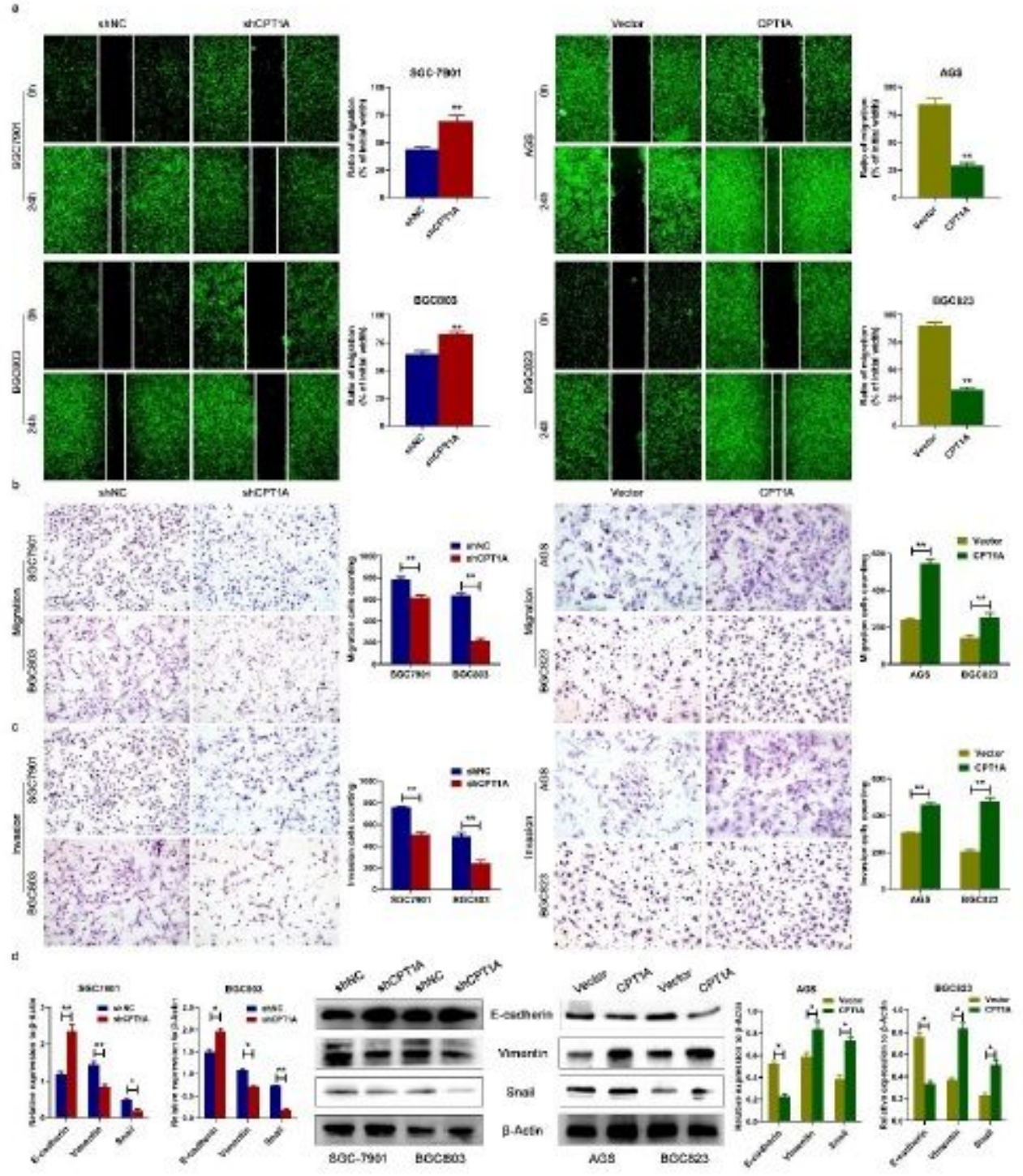

Figure 4

CPT1A enhanced cell migration and invasion in human GC cells. (a). Monolayer wound healing assay was explored the migration abilities of GC cells with CPT1A overexpressed or silenced $(\times 100)$. (b-c). Transwell cell migration (b) and invasion (c) assays tested that CPT1A promotes both cell migratory and invasive capacities in GC cells. The stained cells were counted from four selected fields randomly. Representative images and quantitative results of cell migration and invasion were shown $(\times 100)$. (d). Western blot analysis of EMT marker (E-cadherin, Vimentin and Snail) in indicated cells with overexpressed or silenced of CPT1A. $\beta$-Actin was used as a loading control. Data are represented as mean \pm standard error of mean of at least three independent experiments, ${ }^{*} \mathrm{P}<0.05 ;{ }^{*} \mathrm{P}<0.01$. 


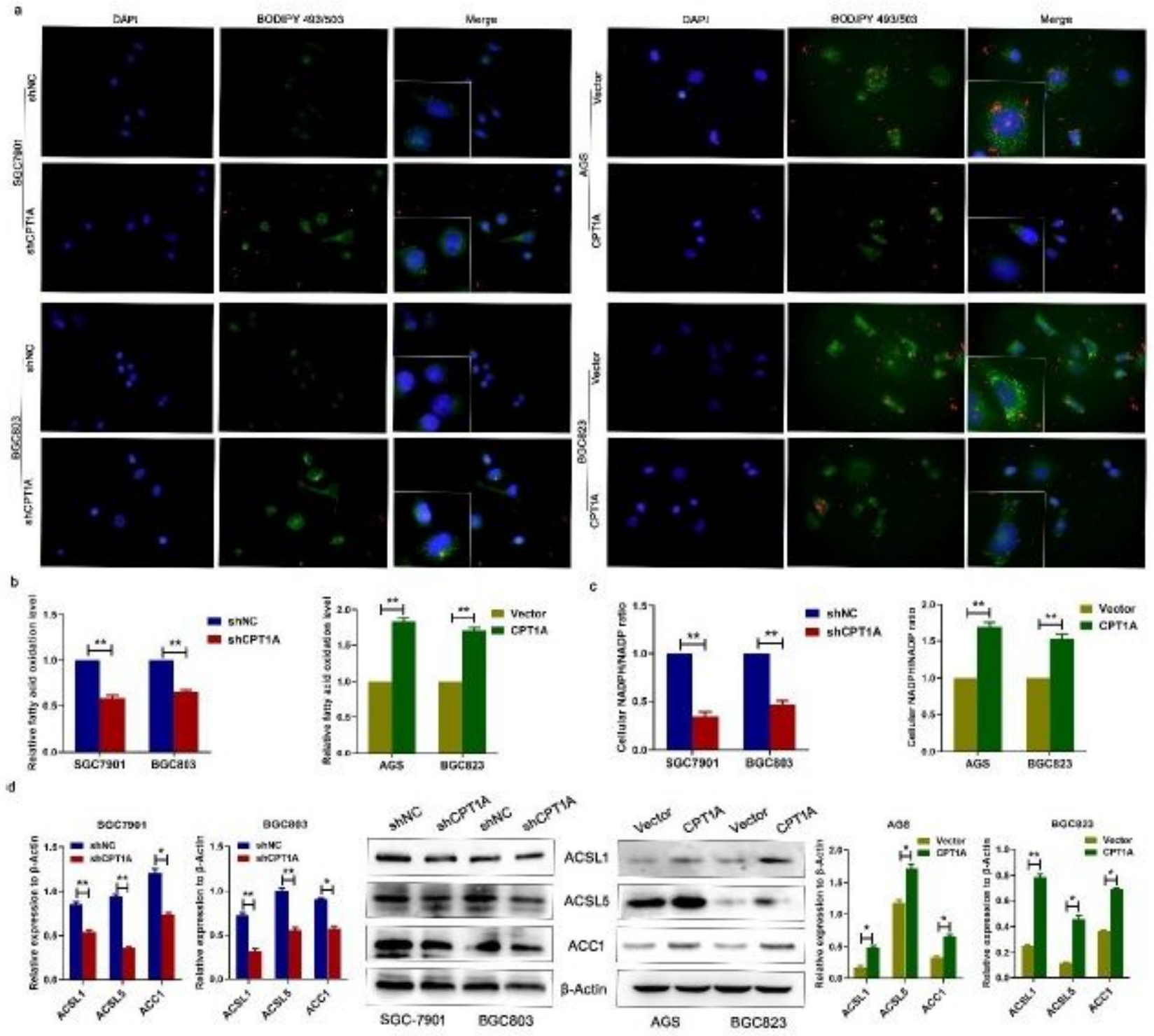

Figure 5

CPT1A enhanced the fatty acid $\beta$-oxidation (FAO) in human GC cells. (a). To indicate fat droplet deposit by Bodipy 493/503 staining in CPT1A-treated GC cells $(\times 200 ; \times 400)$. (b-c). The level of FAO (b) and The NADPH/NADP+ ratio (c) in CPT1Asilenced and CPT1A-overexpressed GC cells. (d). Western blot analysis of FAO marker (ACSL1, ACLS5, ACC1) in indicated cells with overexpressed or silenced of CPT1A. $\beta$-Actin was used as a loading control. Data are represented as mean \pm standard error of mean of at least three independent experiments, ${ }^{\star} \mathrm{P}<0.05$; ${ }^{\star}{ }^{*} \mathrm{P}<0.01$. 
a
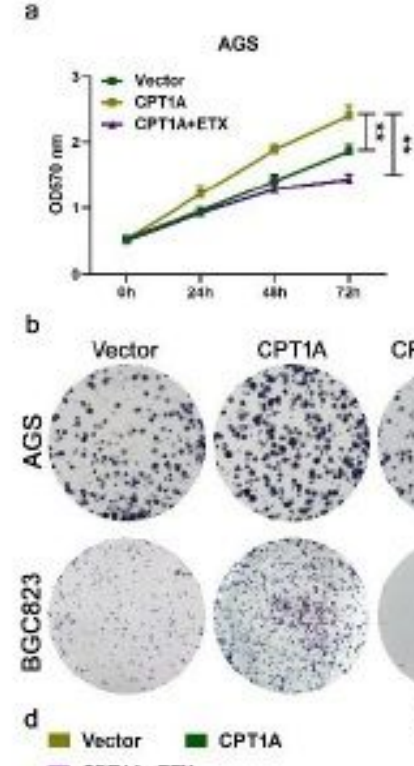

- CPT1A.ETX

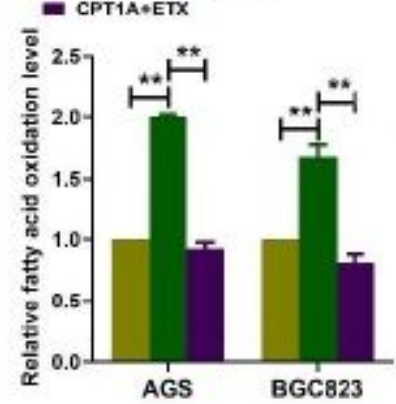

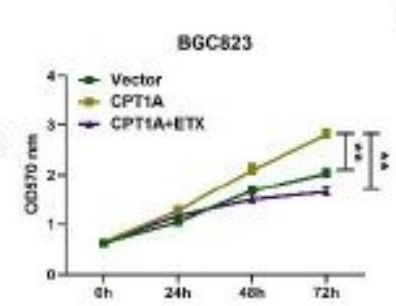

CPT1A+ETX

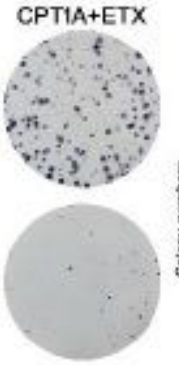

e
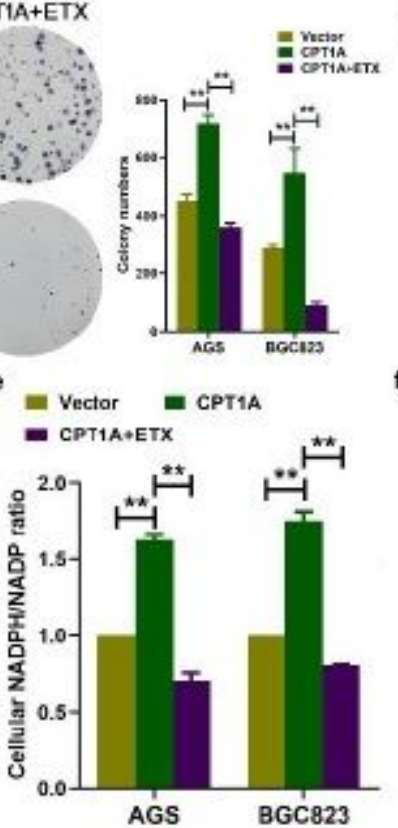

Vector CPT1A CPT1A+ETX

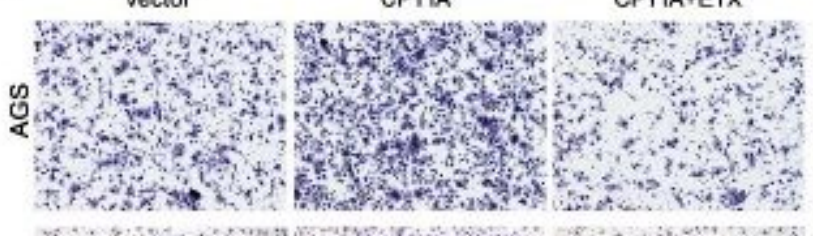

สี

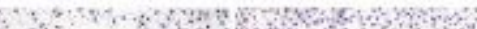

๑

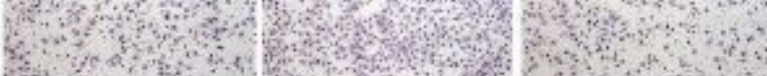

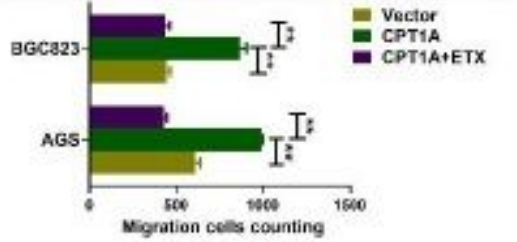

f

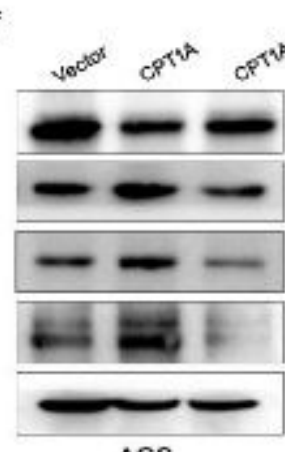

AGS not

E-cadherin

Vimentin

ACSL1

ACC1

$\beta$-Actin

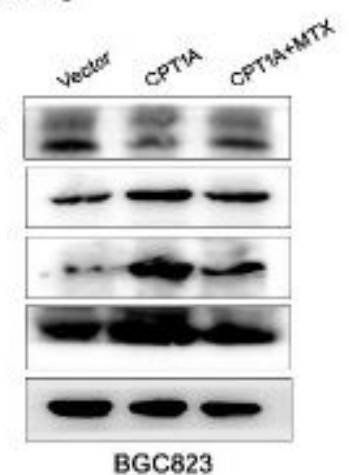

Figure 6

Inhibition of FAO attenuates CPT1A-induced proliferation and migration in GC cells. (a). Cell viability of CPT1A-overexpressed GC cells treated by Etomoxir (ETX) for 24, 48 and $72 \mathrm{~h}$ was measured by MTT. (b). Representative images (left) and quantification (right) of colony formation assay for the ETX-inhibited effects in CPT1A-overexpressed GC cells. (c). Representative images (left) and quantification (right) of Migration assay for the ETX-inhibited effects in CPT1A-overexpressed GC cells. (d-e). The level of FAO (d) and The NADPH/NADP+ ratio (e) for the ETXinhibited effects in CPT1A-overexpressed GC cells. D. Ecadherin, Vimentin, ACSL1 and ACC1 were determined by western blot after exposure to ETX for $48 \mathrm{~h}$ in CPT1Aoverexpressed GC cells. $\beta$-Actin was used as a loading control. Data are represented as mean \pm standard error of mean of at least three independent experiments, ${ }^{*} P<0.05 ; * \star P<0.01$. 


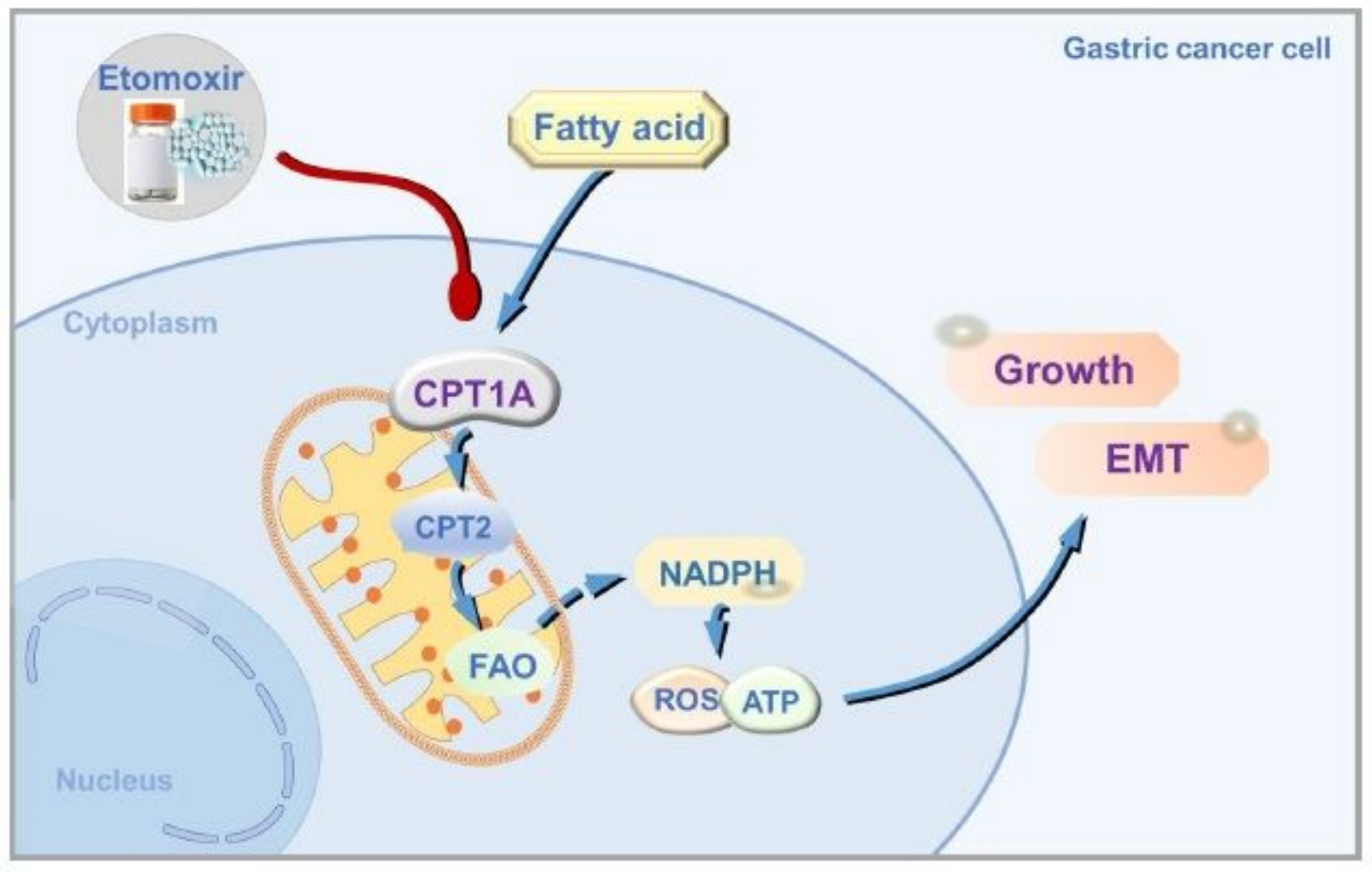

Figure 7

Schematic pathways of CPT1A mediated growth and EMT in breast cancer cells due to increased mitochondrial FAO metabolism.

\section{Supplementary Files}

This is a list of supplementary files associated with this preprint. Click to download.

- Table.pdf

- SupplementaryMaterial.pdf 